\title{
Evoluer hors des enclos
}

\author{
Gérard Authelain
}

\section{(2) OpenEdition}

Journals

Édition électronique

URL : http://journals.openedition.org/trema/868

DOI : $10.4000 /$ trema. 868

ISSN : 2107-0997

\section{Éditeur}

Faculté d'Éducation de l'université de Montpellier

\section{Édition imprimée}

Date de publication : 1 décembre 2005

Pagination : 33-42

ISSN : 1167-315X

\section{Référence électronique}

Gérard Authelain, «Evoluer hors des enclos », Tréma [En ligne], 25 | 2005, mis en ligne le 06 janvier 2010, consulté le 02 mai 2019. URL : http://journals.openedition.org/trema/868 ; DOI : 10.4000/ trema.868

Ce document a été généré automatiquement le 2 mai 2019.

Trema 


\title{
Evoluer hors des enclos
}

\author{
Gérard Authelain
}

1 L'histoire avance rarement de façon linéaire. Les innovations sont le fruit de ruptures autant que d'évolutions en pente douce. Ou du moins les changements importants sont-ils le fait de négociations mettant en jeu des options différentes, voire divergentes. Et c'est parce que cette différence est non seulement acceptée, mais souhaitée, que les paysages les plus enclavés se mettent à se modifier.

\section{Des années qui sont déjà l'histoire}

2 La création des CFMI n'a pas failli à la règle. Déjà dans les années 70, à la faveur du climat contestataire déclenché en 1968, un certain nombre de principes concernant l'éducation en général avaient été mis à mal. L'enseignement de la musique n'avait pas échappé à ce bouillonnement. Nombre d'écoles de musique se voyaient reprocher un élitisme écartant la population d'un accès aux pratiques instrumentales, les deux années de solfège obligatoires étant entre autres un goulet d'étranglement décourageant un grand nombre d'élèves.

3 L'enseignement général n'était pas épargné. Certes les élèves - maîtres des écoles normales bénéficiaient d'un enseignement musical conséquent, mais ces mêmes étudiants se trouvaient écartelés avec un contenu d'enseignement un peu décalé par rapport aux esthétiques en vigueur (on en était alors aux grandes heures de la pop). Et surtout, ils lisaient Carl ROGERS, Ivan ILLICH, Paolo FREIRE, et bien d'autres : ils pensaient que les pratiques artistiques étaient un lieu privilégié pour tenter de nouvelles approches pédagogiques. Ces expériences relevaient plus des réseaux associatifs (et notamment de ceux plus ou moins affiliés à l'éducation populaire) que des organismes institutionnels patentés.

4 Un peu partout en France se mirent à éclore des initiatives où des mots tels qu'invention musicale, corps sonores, jeux vocaux, improvisation, enregistrements, constructions d'instruments, rencontres entre musique et arts plastiques, théâtre musical, etc., tinrent lieu de marque identitaire. Ces pratiques se développèrent souvent ailleurs qu'à l'école, 
mais progressivement aussi dans le cadre scolaire grâce notamment à des instituteurs et institutrices affiliés à des mouvements tels que le GFEN et l'école moderne (Freinet).

De toute évidence, ces actions, portées avec passion par des animateurs musicaux n'ayant reçu de mandat officiel de personne et par des enseignants désireux de briser les carcans trop étroits dans lesquels ils ne se reconnaissaient plus, ne pouvaient que susciter en regard de vives critiques, parfois assassines. Mais l'enjeu du débat dépassait largement quelques initiatives qu'on aurait pu assimiler à un prurit adolescent ou à une effervescence de jeunesse en mal d'originalité. Les questions soulevées étaient d'importance, et faisaient l'objet d'affrontements se déroulant à l'échelon de l'hexagone, aussi bien sur les ondes de la radio nationale que dans les multiples rencontres à caractère plus ou moins militant.

Cet enjeu pouvait se résumer clairement : il fallait « libérer la musique », comme l'avait titré un livre qui ne laissa pas indifférent à sa parution. Il fallait surtout en ouvrir l'accès à tous, (et on comprend que l'école primaire, lieu de passage obligé de toute la population enfantine du pays était la visée de cette opération), en incluant toutes les musiques, les plus contemporaines aussi bien que les plus traditionnelles, et en mettant en jeu une pédagogie qui ne soit pas seulement la transmission de notions et de techniques à apprendre.

7 La force de ce mouvement ne fut pas seulement le fait d'une convergence d'intérêt pour ce type de recherche dans le cadre de l'école primaire. Certaines écoles de musique enclenchèrent également des expériences novatrices qui contribuèrent grandement à donner crédit aux multiples initiatives, y compris à celles qui pouvaient de fait être plus le résultat de bons sentiments que de réelle compétence artistique. Ces quelques établissements d'enseignement spécialisé ont permis d'opérer un saut qualitatif désormais pris en compte dans le dialogue entre le ministère de la Culture et le ministère de l'Education nationale.

\section{Une action concertée}

8 C'est dans ce contexte qu'en 1981 Monsieur André DUBOST, qui avait justement développé, dans le conservatoire d'Yerres qu'il dirigeait, un certain nombre d'initiatives incluant les préoccupations d'un développement des pratiques musicales dans le cadre de l'enseignement général, fut invité au ministère de la Culture à proposer le déploiement d'une telle politique à l'échelle de la nation. Des réunions nombreuses furent alors engagées avec l'Education nationale : cette dernière aurait préféré, de son côté, miser sur l'accroissement de la formation musicale des instituteurs plutôt que sur l'entrée dans l'école d'éléments extérieurs à son institution. Mais le mouvement des idées avait fait son chemin.

9 Certaines contradictions étaient insolubles pour l'heure, ainsi la constitution d'un corps d'instituteurs spécialisés sur le modèle d'autres pays européens n'apparaissait pas souhaitable. Il était impossible financièrement d'accroître de façon significative le temps de formation musicale dans les écoles normales. Devant la nécessité de prendre en compte une situation de plus en plus critique sur le niveau de l'enseignement musical dans l'école élémentaire, bon gré mal gré l'Education nationale accepta les CFMI, mais avec une réserve qui n'a pas disparu chez certains responsables, depuis la rue de Grenelle 
jusqu'aux inspections départementales, dont on verra plus loin la manifestation et les raisons.

10 Neuf Centres de formation furent ainsi ouverts à partir de 1984. On peut admirer encore aujourd'hui la dose de clairvoyance et de courage nécessaire aux responsables pour mettre sur pied une collaboration que la plupart, à l'époque, vouaient à l'échec. En effet les CFMI furent créés sous double tutelle, Culture et Education nationale, obligeant tous les acteurs, responsables ministériels aussi bien qu'étudiants se préparant à leur nouveau métier, à jouer le jeu de la compréhension mutuelle, du partage des responsabilités dans la conduite de l'éducation artistique, du respect des compétences réciproques et des fonctions propres à chacun.

11 Les CFMI sont encore aujourd'hui sous cette double tutelle, malgré les tentatives récurrentes de les placer sous responsabilité d'un seul ministère : ce serait tellement plus simple, entend-on encore aujourd'hui, s'il étaient sous l'égide du seul ministère de la Culture, ou de la seule Université, ou si les musiciens intervenants étaient rattachés au personnel de l'Education nationale. Car il est vrai qu'au cours de leur brève histoire, les tentatives de normalisation (entendons par là une remise dans des formats institutionnels connus) n'ont pas manqué : essais de rattachement aux IUFM, volonté de diminution du quota d'heures de formation pour des motifs budgétaires, coupes sévères dans le budget, réduction des temps de stages, globalisation des cours avec d'autres organismes universitaires, etc.

12 A chaque menace, il a fallu défendre pied à pied l'intuition novatrice des fondateurs, et curieusement, 20 ans après la création et la reconnaissance unanime des CFMI, de nombreux problèmes demeurent et ne paraissent pas en voie de règlement immédiat. C'est probablement la nécessité d'une vigilance permanente qui fait que, malgré la diversité des 9 établissements, la solidarité entre eux a toujours été totale pour maintenir et faire vivre l'intuition d'origine.

\section{Une formation évolutive sur un socle stable}

13 L'originalité de la formation proposée vient de ce qu'elle est à la fois exigeante dans ses objectifs et souple dans ses modalités. Un certain nombre d'orientations sont fixées de manière à fournir une ossature (plus qu'un cadre) stable, avec un propos clairement énonçable, à partir de laquelle de multiples ramifications peuvent se greffer avec une importance plus ou moins grande selon les lieux, les époques, les besoins, les possibilités.

\section{III.1. Une formation réellement professionnelle}

S'agissant de la charpente, l'une des premières options à laquelle tiennent les établissements et qu'ils ne sont pas prêts à voir modifier, est le temps de formation (1500 heures sur deux années) réparties en $2 / 3$ de cours, ateliers, travaux personnels, et $1 / 3$ de stages sur terrain avec suivi régulier de la part des responsables. En d'autres termes, les étudiants, tout au long de leur scolarité, ont un minimum de 500 heures hors murs, en situation de travail identique à celle qu'ils connaîtront lorsqu'ils seront professionnels, dans des écoles qui sont de même configuration que celles de leur future affectation.

L'aller - retour régulier entre les cours et le stage sur terrain permet une maturation, une mise à distance permanente des acquis théoriques et de l'expérimentation «grandeur 
nature ", et ce avec le soutien des responsables., certes, mais aussi entre étudiants. Car l'une des orientations tout aussi fondamentales des CFMI est de préparer les étudiants au travail en collaboration, avec les enseignants, certes, mais aussi une multitude d'autres partenaires, y compris leurs propres collègues.

\section{III.2. Un métier de collaboration}

L'originalité du métier, en effet, est de travailler non pas seul avec un groupe d'enfants, mais de le faire dans un cadre (la classe) où le responsable permanent et référent pour les enfants est le maître (instituteur ou professeur des écoles). Le musicien intervenant est un intervenant non pas étranger ou extérieur, mais associé. Pour cette tâche complexe d'éducation musicale (qui n'est pas un simple éveil ou une seule sensibilisation, mais bien vraiment une tâche mêlant pratique et initiation), deux personnes sont invitées à travailler de concert avec les enfants. Cela a obligé à redéfinir, bon gré mal gré, les positions des uns et des autres, et ce de manière un peu moins sommaire que ne le souhaiteraient les démons de la facilité ou des positions acquises.

Parmi les réorientations opérées se trouve celle de la polyvalence de l'instituteur. Il a fallu mettre en brèche la façon étriquée de concevoir ce dogme intangible dans la philosophie habituelle (l'instituteur est capable de faire la musique dans sa classe, et l'intervenant « extérieur » n'est souhaitable que pour certaines actions ponctuelles et très spécialisées). Il a fallu faire comprendre que la polyvalence n'est pas une "polytalence » (c'est-à-dire que l'instituteur saurait tout faire avec un égal talent), mais une responsabilité pédagogique autrement importante et pour le coup crédible: il est le référent essentiel dans sa classe. Au besoin il s'entoure de collaborations pour des secteurs d'activités plus spécialisés, mais il garde la responsabilité pédagogique en étant auprès des enfants celui qui leur permet de se construire de manière structurante à travers les divers apports, ceux qu'il ne saurait déléguer et ceux pour lesquels il bénéficie de la présence d'intervenants associés et formés à ce partenariat.

\section{III.3. Une compétence musicale}

La troisième orientation fondamentale est de réserver la formation (et donc le métier) à des musiciens ayant une solide formation de base et pouvant faire état d'une pratique instrumentale ou vocale. A ces musiciens, il est alors proposé une formation complémentaire les ouvrant à l'ensemble du champ musical. Outre l'élargissement culturel sur le plan artistique et le développement de la voix au plan personnel et collectif, sont développées les démarches pédagogiques faisant appel à la pratique d'ensemble, à l'inventivité et l'imagination, aux technologies d'assistance à la création musicale. De même sont abordées, à travers des modules d'administration et de gestion, les modalités de travail avec des élus, des collectivités territoriales, des centres culturels, etc.

Un socle est ainsi formé par les trois points d'appui développés

ci-dessus :

- une formation s'adressant à des musiciens confirmés,

- un apprentissage du travail en collaboration avec d'autres, et tout particulièrement avec les enseignants, ainsi qu'avec les autres partenaires culturels du lieu, 
- une formation dont le tiers du temps se déroule en stages sur terrain.

\section{Le constat d'une évolution}

Lorsqu'on compare les programmes des études des premières années et ceux d'aujourd'hui, il est manifeste qu'une réelle évolution s'est produite, la raison des modifications étant généralement liée à l'exercice professionnel. Mais la véritable évolution des CFMI n'est pas dans les mutations qu'ils ont opérées sur la façon de conduire un cursus d'études : elle est surtout dans ce que les musiciens intervenants euxmêmes ont modifié dans le paysage de l'éducation et de la pratique musicale. Autant de situations inventées que les CFMI ont avalisées et pour lesquelles ils ont adapté leur programme de formation.

21 Les transformations se sont manifestées sur plusieurs plans : celui de l'école, celui des écoles de musique, celui des pratiques culturelles dans les communes, celui du statut de musicien-enseignant.

\section{IV.1. Une collaboration bénéfique pour tous}

A l'école, les maitres ont découvert un auxiliaire précieux pour mener des parcours artistiques pour lesquels ils n'étaient pas formés. Ils se sont aperçus à la fois qu'ils n'étaient pas supplantés dans leur rôle (comme le craignaient et le craignent encore tant d'inspecteurs), et qu'ils n'étaient pas non plus dispensés de participer activement à la séance musicale sous la forme qui leur convenait le mieux. Autrement dit, alors que se multipliaient toutes les précautions pour l'intervention d'un musicien «dumiste » dans une classe (circulaires limitant le nombre d'heures d'intervention à 10 ou 12 heures sur une année, obligations d'un agrément de projet facilement confondu avec un agrément de personne, tracasseries administratives multiples agaçant jusqu'à certains maires), le mouvement de collaboration s'accentuait néanmoins et inexorablement, et reléguait au niveau d'un sectarisme désuet les tentatives de réaction et de crispation sur l'ancien.

3 Aujourd'hui les professeurs d'IUFM et les CPEM reconnaissent pour la plupart que les professeurs des écoles bénéficient par voie de rebond des activités menées en commun avec eux par les musiciens intervenants. A tel point que la formation continue se développe de plus en plus, sous des formules diverses, de manière conjointe, les uns et les autres s'apportant leurs richesses mutuelles pour leur plus grand bien personnel et celui des enfants. L'hostilité n'a pas totalement disparu, dans la mesure où les responsables de l'institution sise rue de Grenelle s'obstinent à ne pas vouloir reconnaitre officiellement l'évolution, mais ce silence désormais fait tache, surtout quand la méfiance s'appuie sur un discours de moins en moins tenable.

\section{IV.2. L'école de musique associée}

Beaucoup de musiciens intervenants sont employés par des écoles de musique. Grâce à leurs activités hors établissement (puisqu'ils vont dans les écoles de quartier), et tout en faisant partie de l'équipe des professeurs, ils réalisent ainsi un premier palier de ce qui est souvent demandé aux écoles de musique : une présence plus effective dans les quartiers. En effet le travail du musicien intervenant est bien de déployer une initiation musicale 
pour tous. Mais cette initiation ne se limite pas à une démarche d'enseignement, elle va jusqu'à mettre en œuvre les conditions d'une authentique pratique musicale collective. Les concerts menés par les enfants se sont multipliés, et ne sont pas seulement des auditions de fin d'années permettant de jauger les progrès d'une année. Ils sont considérés comme de vraies fêtes musicales, souvent avec des répertoires difficiles et très novateurs (on pense ainsi au répertoire fourni par les Editions Mômeludies) ou des pièces issues d'un travail de création avec la classe.

Pour accompagner ces enfants dans leurs prestations publiques, le musicien intervenant fait appel aux instrumentistes de l'école de musique dont il dépend, que ce soient les professeurs ou les grands élèves en fonction de la difficulté des pièces. Ainsi se développe une collaboration entre enseignement général et enseignement spécialisé qui ne se cantonne pas aux seuls aspects didactiques, mais se présente bien comme une pratique musicale commune.

Il est par ailleurs significatif (les annonces professionnelles de l'hebdomadaire Télérama en font foi) que les écoles de musique cherchent fréquemment à embaucher un dumiste dont une partie du temps sera consacré à des activités sur les quartiers hors scolaire (musiques actuelles, ateliers d'éveil, etc.). La raison en est simple : leur formation les rend particulièrement prêts à s'adapter et à adapter leur fonction aux situations les plus diverses et les moins prédéfinies.

\section{IV.3. Une action culturelle}

Les maires de nombreuses communes, rurales ou urbaines, n'ont pas tardé à saisir combien le profil d'un professionnel issu d'un CFMI pouvait répondre en partie à leurs souhaits. Depuis longtemps le musicien intervenant est identifié comme un acteur culturel local indispensable, et pas seulement parce qu'il répond au désir des parents souhaitant une initiation musicale générale pour leur enfant dans le cadre de son cursus scolaire.

Ce musicien, selon sa personnalité, est sollicité pour diriger une chorale, ou pour diriger une harmonie, ou pour intervenir dans un établissement de handicapés, ou pour quelques séances dans des crèches, ou pour venir en soutien d'activités avec des adolescents dans un établissement d'éducation populaire, etc. La disponibilité notée plus haut dans le cadre des écoles de musique se retrouve ici au plan des collectivités territoriales. Les CFMI n'ont pas manqué d'alerter les maires sur cette possibilité de développer l'action culturelle tout en développant dans la formation des futurs dumistes cette nouvelle mission possible.

\section{IV.4. Des musiciens identifiés comme artistes}

Enfin la fonction de musicien intervenant, dans la mesure où les tâches d'enseignement ne supplantent pas le statut de musicien, engagent celui-ci par goût personnel (et non par obligation légale) à ne pas abandonner la pratique instrumentale ou vocale personnelle. Nombreux sont ceux qui choisissent de n'intervenir qu'à mi-temps dans les écoles élémentaires pour consacrer le reste du temps à une activité musicale personnelle, au sein des groupes les plus divers (ensemble instrumental, chanson, spectacles jeune public, composition, etc.) 
30 Il était de bon ton, à une époque, de penser que musicien intervenant dans une école signifiait une qualité moindre au niveau instrumental. Dans la mesure où de nombreux musiciens intervenants ont fait le choix d'une activité de spectacle rémunérée, et où ils en vivent fort bien, les vieux clichés sont amenés à s'auto - détruire. Le temps où l'on conseillait à une jeune clarinettiste de CNR de se diriger du côté de l'animation scolaire (sous-entendu parce qu'elle n'avait aucune chance dans le cercle fermé des instrumentistes reconnus) est définitivement clos. Certains se sont produits sur les plus grandes scènes (Printemps de Bourges, Francofolies), d'autres font partie d'ensembles renommés (Ensemble orchestral contemporain, solistes de chœurs professionnels), et on pourrait multiplier ainsi les exemples d'une intense vie artistique d'un grand nombre de dumistes.

\section{Une évolution à évaluer}

31 Au terme de ce survol, le lecteur peut se demander en quoi les derniers paragraphes concernent l'Education nationale. C'est ce à quoi il faut tenter de répondre maintenant.

\section{V.1. Une école qui élargit son enseigne}

Il a été suffisamment dit combien le métier de musicien intervenant, officialisé par les deux ministères, a amorcé une mue dans la façon de comprendre les enseignements artistiques dans le premier degré, et plus encore dans la manière de les mettre en œuvre. Le dogme de la polyvalence est battu en brèche dans ses acceptions traditionnelles et sera de moins crédible sous sa formulation ancienne : les orientations données aux IUFM ne paraissent pas devoir multiplier le nombre d'heures de formation musicale des futurs professeurs des écoles, et les attentes de la population en matière artistique sont plus exigeantes qu'il y a un demi siècle ou un siècle.

En même temps, cette collaboration demeure une volonté et son exercice ne pourra jamais totalement être circonscrit par des circulaires ou des règlements. L'école primaire fait la preuve qu'elle est certes un lieu où sont dispensés les enseignements fondamentaux irremplaçables pour l'acquisition des compétences dont aura besoin le futur citoyen. Mais elle ressemble aussi à quelque chose qui s'apparente à un centre culturel, le seul encore vaillant dans certains quartiers devenus parfois inhumains, le seul qui apporte aux enfants un espace de pratique autre que le formatage qui lui est conféré par la rue ou la télévision.

34 La musique à l'école est un ensemble d'activités qui apportent un temps de gratuité et de partage, un signe de collaboration entre adultes et de découverte des enfants sous d'autres angles, un parcours qui mêle plaisir et exigence, découverte d'un patrimoine et découverte de soi. Elle offre un répertoire qui ne se limite pas à quelques chansonnettes, une marseillaise et un chant du départ - pour aller où? -. Elle apporte une contribution pour que l'école demeure ce lieu où les enfants continuent à venir en sachant que c'est leur première (et souvent leur dernière) chance de se réaliser au sein d'un groupe en reculant les frontières de leur milieu naturel.

Les enseignements artistiques ne semblent pas avoir préoccupé outre mesure les rédacteurs du document de la commission THELOT. Et pourtant, tant que le ministère de la Culture et de l'Education nationale continuent à vouloir ensemble un type original 
d'intervention à l'école en matière musicale (et qui n'a pas d'équivalent dans les autres pays européens), il est important que soit rappelé le rôle unique que peut jouer l'école.

\section{V.2. L'école dans son rapport avec les autres instances culturelles} les différentes cultures: celles de l'enfant avec celles de parents qui n'ont pas eu les mêmes chances, celles d'enfants dont le patrimoine familial encore vivant ne sort guère des cercles communautaires. Le brassage peut, grâce à l'action musicale, être l'occasion d'un échange qui ne soit pas de simple coexistence, mais de réelle rencontre. Ce n'est ni simple ni gage de réussite, mais c'est au moins une tentative qui vaut d'être menée. En ce sens là aussi l'école est le centre d'un vivier culturel qu'un musicien intervenant, en apportant sa collaboration aux enseignants, peut aider à développer.

\section{En conclusion}

40

Evoluer hors des enclos n'est ni confortable ni sécuritaire. En fait parler d'enclos est une façon d'avouer que certains considèrent leur espace de travail habituel comme une propriété privée, où personne d'autre qu'eux-mêmes n'a droit de regard. Et devant cette frilosité, certains sont tentés d'abattre les murs et de pénétrer par force sur un territoire où ils n'ont pas été invités. 
41 Les CFMI ont été une réponse à cette double tentation. L'école est le lieu privilégié d'une initiation à la musique, parce qu'elle est le passage obligé de tous les enfants d'une nation et qu'une petite minorité d'entre eux seulement aura accès à l'enseignement spécialisé. En même temps elle n'est pas le champ de toutes les expériences, si généreuses soientelles: travailler en collaboration avec des enseignants réclame une formation et un apprentissage du travail en commun, dans le respect des compétences mutuelles et des missions réciproques.

42 Si la réforme de l'école fait partie des thèmes sempiternellement remis sur la table de travail, la façon dont s'est développé un mode de travail commun dans l'école et les conséquences qui en apparaissent pour le rôle et le crédit de l'école, peuvent sans doute aider à la réflexion commune. Il n'est pas interdit aux commissions réfléchissant sur le rôle futur de l'école de s'apercevoir que, malgré tout, l'histoire avance.

\section{RÉSUMÉS}

La création des Centres de formation des musiciens intervenants en 1984, sous la double tutelle des ministères de la Culture et de l'Education nationale, constitua une avancée considérable pour l'enseignement musical primaire en France. Gérard Authelain décrit en outre les spécificités du cursus et souligne ses contributions, entre art et pédagogie.

In 1984 the creation of training centres for visiting music teachers, under the supervision of the Ministry of Culture and the Ministry of Education, was a major step forward for music teaching in French primary schools. Gérard Authelain also describes the specificities of this type of training and underlines its contribution, between art and pedagogy.

\section{AUTEUR}

\section{GÉRARD AUTHELAIN}

Ancien directeur du CFMI de Lyon, Université Lumière Lyon II 